\title{
FUZZY TECHNIQUES IN OBJECT BASED MODELING
}

\author{
Laxman Sahoo ${ }^{1}$, Praveen Kumar Shukla ${ }^{2}$ \\ 'Northern India Engineering College, Lucknow, UP, India \\ ${ }^{2}$ Department of Information Technology, Northern India Engineering College, Lucknow, UP, India \\ E-mail : ${ }^{1}$ laxmansahoo@yahoo.com, ${ }^{2}$ praveenshuklaniec@yahoo.co.in
}

\begin{abstract}
Inexact knowledge engineering information plays vital role in decision making capabilities within latest areas of information technology. However, this information is often vague or ambiguous and very difficult to represent in implementing the application softwares, but this problem can be handled by modeling imprecision and uncertainty in conceptual data models. In this paper, we have investigated the methods of designing and implementing fuzzy information with different object modeling techniques to handle uncertain data. Also, we have described the different levels of fuzziness with all object modeling representations.
\end{abstract}

Key words: Aggregation, Association, Generalization, Fuzzy, Object Model, Possibility Theory.

\section{INTRODUCTION}

Databases have gone through the development from hierarchal and network databases to relational databases. When the databases are used for CAD/CAM, knowledge based systems, multimedia and Internet; so many limitations have been encountered in the relational databases. Hence, ER data models [1], object oriented data models and logic models have been proposed. In traditional models, we are assuming that data stored is known, accurate and complete. But what about the uncertain data? Now, let us consider the following concept of data uncertainty. Five basic types of imperfection have been introduced in this research paper. These are: 1.Inconsistency 2.Imprecision 3. Vagueness 4. Uncertainty 5.Ambiguity [2]. Inconsistency is a kind of semantic conflict when some aspect of the real world is irreconcilably represented more than once in a database or in different databases. In imprecision and vagueness, the value attributed to an attribute or the interpretation assigned to the concept, is known to come from a given interval or a set of values but we do not know exactly which one to choose at present. Uncertainty refers to those situations, in which we can apportion some, but not all, of our belief to the fact that an attribute took a given value or a group of given values. In ambiguity some elements of this model lack complete semantics, leading to several possible interpretations. Vagueness and uncertainty are generally modeled with fuzzy sets and possibility theory $[3,4]$. The fuzzy information is also handled with relational database concepts. Recent efforts have extended these results to object oriented databases by introducing the related notions of classes, generalizations, specialization and inheritance $[5,6,7,8]$. The uncertainty is handled at data level and conceptual model level both $[9,10,11]$.

The additional things that are to be captured in object oriented database methods are items triggers, indexes and various types of constraints directly as part of the diagram. Object oriented concepts are being applied to data modeling.[12,13] More recently, these concepts are used to model XML conceptually [14]. One thing lacking in the object oriented databases modeling can be generalized as the need to handle imprecision and uncertain information. Although imprecise and uncertain information exists in knowledge engineering and database systems, and have extensively been studied. In this paper, the fuzzy implementations are derived with object modeling concepts like class, generalization, aggregation, association etc.

\section{FUZZY CLASS}

We can implement a class by two methods named extensional and intensional implementation. In extensional implementation of the class, the class is defined by the list of objects, but in intensional implementation of the class, the class is defined by a set of attributes with their admissible values.

First, it is possible that some objects are fuzzy ones, which have similar properties. A class defined by these objects may be fuzzy. These objects belong to the class with a membership degree of $[0,1]$. Second, the domain of an attribute may be fuzzy and fuzzy class is formed.

Third, the sub class produced by a fuzzy class using specialization and super class produced by some classes using generalization, in which at least one class is fuzzy, are also fuzzy.

Now in the context of the class, we can define three levels of the fuzziness in the classes: [15]

1. Fuzziness in terms of, class belongs to the data model.

2. Fuzziness related to whether some instances are 
instances of a class, even though the structure of the class is crisp.

3. Fuzziness in attribute values of the instances of the class.

\section{A. First level of fuzziness}

In the first level of the fuzziness, we write the following syntax to present the class name or attribute in the class.

\section{CLASS NAME WITH $m$ DEGREE}

where $0 \leqslant m \leqslant 1[16,17]$

Like, for a class in a data model, we can write the syntax "Person WITH 0.8 DEGREE". The class will not be declared having degree 0 and 1 . For example, we do not write the syntax like [12], "PERSON WITH 1 DEGREE" or "PERSONWITHODEGREE"

\section{$B$. Second level of fuzziness}

We must indicate the degree of fuzziness to which an instance of the class belongs to the class. For this, we introduce a special attribute $\lambda$ to represent the instance membership degree to the class.

\section{Third level of the fuzziness}

The fuzziness in terms of attribute's value domain, we write the following syntax like, "JOB WITH 0.5 DEGREE". But in the second case, when an attribute may take fuzzy values namely, its domain is fuzzy; we introduce a different syntax like "FUZZYAGE".

Such fuzzy classes will be represented by dashed outline rectangle.

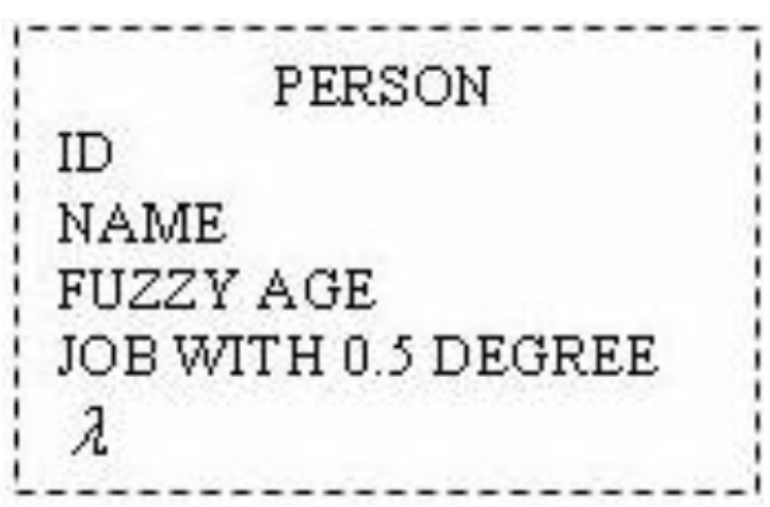

Fig .1 Fuzzy class

\section{FUZZY GENERALIZATION}

A class produced from a fuzzy class must be fuzzy. Hence, sub-class and super-class relationship is fuzzy. That means, a class is the subclass of another class with membership degree of $[0,1]$ at that time. Now we are considering the two methods to determine the subclass - super class relationship.

\section{A. Fuzzy generalization considering first level of fuzziness in the classes}

Assume that $X$ and $Y$ are the two classes having $X$ WITH mem_XDEGREE and YWITH mem_YDEGREE.

Then $Y$ is the subclass of $X$ if $(\mathrm{Ae})\left(\beta \xi_{\gamma}(\mathrm{e}) \xi_{\chi}(\mathrm{e})\right)^{\wedge}(\beta \leqslant$ mem_Y $\leqslant$ mem_X) Consider a fuzzy super class $X$ and its fuzzy subclasses $Y 1, Y 2, Y 3 . . . . . Y n$ with instance membership degree $\mu_{x}, \mu_{\gamma_{1}}, \mu_{\gamma_{2}} \ldots \ldots \ldots \ldots \mu_{\gamma_{n}}$, which may have degree of membership mem_X, mem_Y1, mem_Y2,.............mem_Yn respectively.

Then following relationship is true.

$(\forall \mathrm{e})\left(\max \left(\mu_{\mathrm{Y}_{1}}(\mathrm{e}), \mu_{\mathrm{Y}_{2}}(\mathrm{e}), \ldots \ldots \ldots \ldots, \mu_{\mathrm{Y}_{\mathrm{n}}}(\mathrm{e})\right) \mu_{\mathrm{x}}(\mathrm{e})\right)^{\wedge}$ (max (mem_Y1,mem_Y2,.....,mem_Yn) mem_A)

With intensional implementation of the class, no object is available. Hence, above method is not feasible.

At this point, we can use the inclusion degree of a class with respect to another class to determine the relationships between fuzzy subclass and fuzzy super class. This was proposed for assessment of data redundancy in fuzzy relational databases $[18,19]$.

The inclusion degree is extended to evaluate the membership degree of an object to a class and further the relationships between the fuzzy subclass and super class.

For example, let $X$ and $Y$ are two fuzzy classes and the degree that $Y$ is the subclass of $X$ be denoted by $\mu(X, Y)$.

The threshold given is $\beta$, and then $Y$ is a subclass of $X$ if $\mu(X, Y) \neq \beta$.

Now consider the following situation, in which we have two classes $X$ and $Y$ as follows:

\section{$X$ WITH mem X DEGREE}

Y WITH mem_Y DEGREE.

Then $Y$ is the subclass of $X$ if $\mu(X, Y) \geqslant \beta) \wedge((\beta \leqslant$ mem_Y $\leqslant$ mem_X $))$

\subsection{Fuzzy generalization considering second level of fuzziness in the classes}

The sub class is a sub class of the super class with membership degree, which is minimum in the membership degree to which these objects belong to the subclass, when these two rules are true.

1. For any fuzzy object, if the membership degree that it 
belongs to the sub class is less than or equal to the membership degree that it belongs to the super class.

2. The membership degree that it belongs to the sub class is greater than or equal to the given threshold.

Formally, let $X$ and $Y$ are the two fuzzy subclasses and be a given threshold. We say $Y$ is a subclass of $X$ if $(\forall e)$ $\left(\beta \Varangle_{y}(e), \bar{F}_{x}(e)\right)$

Here, $e$ is the object instance of the class $X$ and $Y$ in the universe of discourse, $\mu_{\mathrm{X}}(\mathrm{e})$ and $\mu_{\mathrm{y}}(\mathrm{e})$ and are the membership degrees of $e$ to $X$ and $Y$, respectively.

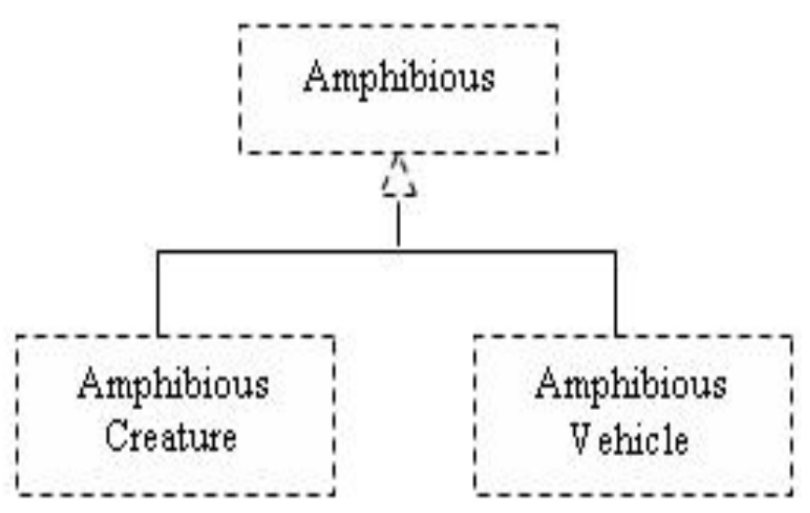

Fig .2 Fuzzy generalization

\section{FUZZY AGGREGATION}

An aggregation captures a whole - part relationship between an aggregate and a constituent part. These constituent parts can exists independently. Therefore, each instance of an aggregate can be projected into a set of instances of constituent parts.

Let $X$ be an aggregation of the constituent parts $Y_{1}$, $Y_{2}, \ldots \ldots$ and $Y_{n}$.

For, the projection of $e$ to $Y_{i}$ is denoted by $\downarrow_{Y_{i} \text {. Then, we }}$ have $\left(\mathrm{e} \downarrow_{Y_{1}}\right) \in Y_{1},\left(e \downarrow_{Y_{2}}\right) \in Y_{2}$, ... $\left(\mathrm{e} \downarrow_{Y_{n}}\right) \in Y_{n}$

A class aggregated from fuzzy constituent parts must be fuzzy. If the former is still called aggregate, the aggregation is fuzzy. Hence, a class is an aggregation of constituent parts with the membership degree of $[0,1]$.

\subsection{Method of determining the fuzzy aggregation}

1. For any fuzzy object, if the membership degree to which it belongs to the aggregate is less than or equal to the membership degree to which its projection to each constituent parts belongs to the corresponding constituent parts [20].

2. The membership degree to which it belongs to the aggregate is greater than or equal to the given threshold [20].

The aggregate is then an aggregation of the constituent's parts with the membership degree, which is minimum in the membership degree to which the projection of theses objects to these constituent parts belong to the corresponding constituent parts.

Let $X$ be a fuzzy aggregation of fuzzy class sets $Y_{1}, Y_{2}$, $\ldots Y_{n}$, with instance membership degree that are $\mu_{x}, \mu_{Y_{1}}, \mu_{Y_{2}}, \ldots \ldots . . . \mu_{Y_{n}}$ respectively.

Given threshold is $\beta$.

$(\forall \mathrm{e})\left(\mathrm{e} \in \mathrm{X} \wedge \beta \leqslant \mu_{X}(\mathrm{e}) \leqslant \min \left(\mu_{Y_{1}}\left(\mathrm{e} \downarrow_{Y_{1}}\right), \mu_{Y_{2}}\left(\mathrm{e} \downarrow_{Y_{2}}\right) \ldots \ldots \ldots . .\right.\right.$.

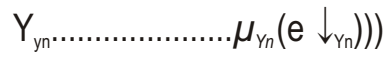

The membership degree that $X$ is an aggregation of class sets $Y_{1}, Y_{2}, \ldots \ldots \ldots Y_{n}$, should be

$\min _{\mu B i(\mathrm{e} \backslash \mathrm{Bi}) \circledast \beta}\left(\mu_{B i}\left(\mathrm{e} \downarrow_{B \mathrm{~B}}\right)\right)(1 \leqslant \mathrm{i} \leqslant \mathrm{n})$

Here, $e$ is an object instance of $X$.

Now we consider first level of fuzziness in above discussed classes.

$X$ WITH mem_XDEGREE

Y1 WITH mem_Y1 DEGREE

Y2 WITH mem_Y2 DEGREE

\section{YnWITH mem_YnDEGREE}

Then $X$ is the aggregate of $Y 1, Y 2, \ldots \ldots \ldots \ldots$ and $Y n$ if

$(\forall \mathrm{e})\left(\mathrm{e} \in \mathrm{X} \wedge \beta \leqslant \mu_{X}(\mathrm{e}) \leqslant \min \left(\mu_{Y_{1}}\left(\mathrm{e} \downarrow_{Y_{1}}\right), \mu_{Y_{2}}\left(\mathrm{e} \downarrow_{Y_{2}}\right) \ldots \ldots \ldots .\right.\right.$. $\left.\left.Y_{Y_{n}}\left(e \downarrow_{Y_{n}}\right) \wedge m e m_{-} Y_{n}\right)\right)$

Here, $\beta$ is the given threshold. When we are implementing the class intentional point of view, we present the use of inclusion degree with fuzzy aggregation relationship [5].

Let $X$ be a fuzzy aggregation of fuzzy class sets $Y 1$, $Y 2, \ldots \ldots \ldots \ldots$....and $Y n$ and $\beta$ is the given threshold. Let the projection of $X$ to $Y i$ be denoted by $X \downarrow_{Y_{\mathrm{i}}}$.Then $\min (\mu(\mathrm{Y} 1$, $\left.\left.\mathrm{X} \downarrow_{Y_{1}}\right), \mu\left(\mathrm{Y} 2, \mathrm{X} \downarrow_{\mathrm{Y}_{2}}\right), \ldots \ldots . . . \mu\left(\mathrm{Yn}, \mathrm{X} \downarrow_{Y_{\mathrm{n}}}\right)\right) \geqslant \beta$

Here, $\mu\left(\mathrm{Y} 1, \mathrm{X} \downarrow_{\downarrow_{1}}\right) \ldots \ldots .(1 \leqslant \mathrm{i} \leqslant \mathrm{n})$ is the degree to which Yi semantically includes $X \downarrow_{Y_{\mathrm{i}}}$.

The membership degree to which $X$ is an aggregation of $Y 1, Y 2, \ldots \ldots \ldots . .$. and $Y n$ is $\min (\mu(B 1, A \downarrow$ $\left.\left.{ }_{\mathrm{B} 1}\right), \mu\left(\mathrm{B} 2, \mathrm{~A} \downarrow_{\mathrm{B} 2}\right), \ldots \ldots . . . \mu\left(\mathrm{Bn}, \mathrm{A} \downarrow_{\mathrm{Bn}}\right)\right)$ 
The above expression can be extended for the situation in which, A, B1, B2,.....Bn may have the first level of fuzziness. Like they may have fuzzy classes with membership degrees as follows:

$X$ WITH mem_XDEGREE

Y1WITH mem_Y1DEGREE

Y2WITH mem_Y2DEGREE

\section{YnWITH mem_YnDEGREE}

Then $X$ is an aggregate of $Y 1, Y 2, \ldots \ldots \ldots \ldots . . . . Y n$ if $\min \left(\mu\left(\mathrm{Y} 1, \mathrm{X} \downarrow_{\left.\mathrm{Y}_{1}\right)}\right), \mu\left(\mathrm{Y} 2, \mathrm{X} \downarrow_{\mathrm{Y}_{2}}\right), \ldots \ldots \ldots\left(\mathrm{Yn}, \mathrm{A} \downarrow_{\mathrm{Yn}_{\mathrm{n}}}\right)\right) \geqslant$ $\beta \wedge$ mem_X $\leqslant$ min $($ Mem_Y1,mem_Y $2, \ldots \ldots \ldots \ldots \ldots . . . .$. mem_Yn))

A dashed open diamond is used to denote fuzzy aggregation relationship.

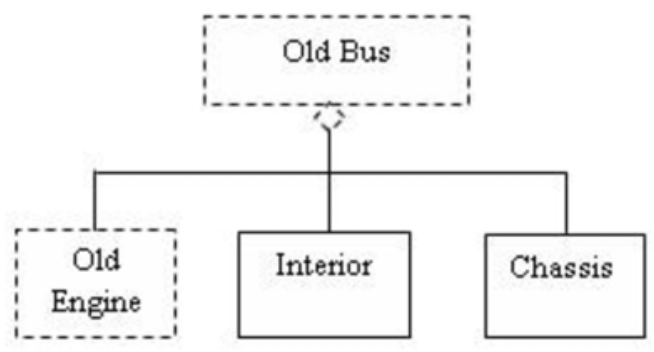

Fig .3 Fuzzy Aggregation

\section{FUZZY ASSOCIATION}

Two levels of fuzziness would be identified in the association relationship.

1. An association relationship fuzzily exists in two associated classes, this association relationship occurs with a degree of possibility.

2. It is possible that it is unknown for certain if two class instances respectively belonging to the associated classes have the given association relationship, although this association relation ship must occur in these two classes [21].

An instance belongs to a given class with a membership degree. Both of the two above fuzziness can be possible simultaneously. First level fuzziness is at class level and second level of fuzziness is at instance level.

We can place a pair of words WITH mem DEGREE \&mem $\$$ after the rolename of association relationship to represent the first level of fuzziness in the association relationship [4].

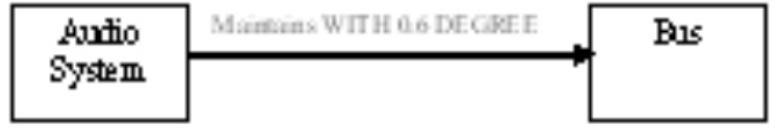

Fig .4 First level of fuzzy association

We use a double line with an arrowhead to denote the second level of fuzziness in the association relationship.

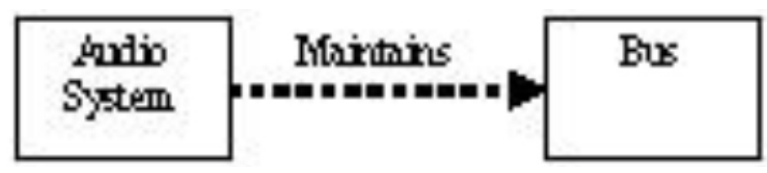

Fig .5 Second level of fuzzy association

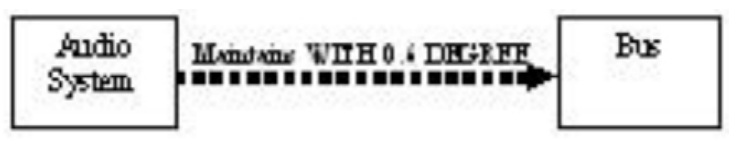

Fig .6 Combining first \& second level of fuzzy association

\section{CONCLUSION}

The fuzzy object modeling is the extended form of the classical object modeling with certain added features. When there is no fuzziness in the universe of discourse, the fuzzy object modeling can be reduced to the classical object modeling. The focus is emphasized on modeling of fuzzy data in the object modeling. In future, the outcome of this paper would be a path for researchers basically on the study of class operations, constraints, and rules in the fuzzy object oriented modeling. In addition, mapping the fuzzy object oriented modeling into object-oriented databases will be main theme of the research.

\section{ACKNOWLEDGEMENT}

We are highly grateful to our Hon'ble Chairman Dr. Akhilesh Das and Vice - Chairperson, Mrs. Alka Das, BBDGEI, for their continuous support, encouragement and guidance to carry out R\&D. Also we would like to express thanks to Mr. R.K. Agarwal, Executive-Director for his suggestions and help.

\section{REFERENCES}

[1] Chen, P. P. (1976). The entity-relationship model: Toward a unified view of data. ACM Transactions on Database Systems, 1(1), 9-36.

[2] Buckles, B. P., \& Petry, F. E. (1982). A fuzzy representation of data for relational database. Fuzzy Sets and Systems, 7(3), 213-226.

[3] Zadeh, L. A. (1965). Fuzzy sets. Information and Control, 8(3), 338-353.

[4] Zadeh, L. A. (1978). Fuzzy sets as a basis for a theory of possibility. Fuzzy Set sand Systems, 1(1), 3-28. 
[5] Bordogna, G., Pasi, G., \& Lucarella, D. (1999). A fuzzy object-oriented data model for managing vague and uncertain information. International Journal of intelligent Systems, 14 , 623-651.

[6] Cross, V., Caluwe, R., \&Vangyseghem, N. (1997). A perspective from the Fuzzy Object Data Management Group (FODMG). In Proceedings of the 1997 IEEE international Conference on Fuzzy Systems, 2, 721-728.

[7] Cross, V., \& Firat, A. (2000). Fuzzy objects for geographical information systems. Fuzzy Sets and Systems, 113, 19-36.

[8] Dubois, D., Prade, H., \& Rossazza, J. P. (1991). Vagueness, typicality, and uncertainty in class hierarchies. International Journal of Intelligent Systems, 6, 167-183.

[9] George, R., Srikanth, R., Petry, F. E., \& Buckles, B. P. (1996). Uncertainty management issues in the object-oriented data model. IEEE Transactions on Fuzzy Systems, 4(2), 179-192.

[10] Gyseghem, N. V., \& Caluwe, R. D. (1998). Imprecision and uncertainty in UFO database model. Journal of the American Society for Information Science, 49(3), 236-252.

[11] Lee, J., Xue, N. L., Hsu, K. H., \& Yang, S. J. (1999). Modeling imprecise requirements with fuzzy objects. Information Sciences, 118, 101-119.

[12] Ambler, S. W. (2000a). The design of a robust persistence layer for relational databases. Retrieved from the World Wide Web: h t t p : / / w w w . a m b y s of t . com / persistenceLayer.pdf

[13] Ambler, S. W. (2000b). Mapping objects to relational databases. Retrieved from the World Wide Web:http://www.AmbySoft.com/mapping Objects.pdf

[14] Conrad, R., Scheffiner, D., \& Freytag, J. C. (2000). XML conceptual modeling using UML.

[15] Zvieli, A., \& Chen, P. P. (1986). Entityrelationship modeling and fuzzy databases. In Proceedings of the 1986 IEEE International conference on Data Engineering (pp. 320-327).

[16] Gyseghem, N. V., \& Caluwe, R. D. (1998). Imprecision and uncertainty in UFO database model. Journal of the American Society for Information Science, 49(3), 236-252.

[17] Marín, N., Medina, J. M., Pons, O., Sánchez, D., \& Vila, M. A. (2003). Complex object comparison in a fuzzy context. Information and Software Technology, 45(7), 431-444.

[18] Ma, Z. M., Zhang, W. J., \& Ma, W. Y. (1999). Assessment of data redundancy in fuzzy relational databases based on semantic inclusion degree. Information Processing Letters, 72(1-2), 25-29.

[19] Ma, Z. M., Zhang, W. J., Ma, W. Y., \& Chen, G. Q. (2001). Conceptual design of fuzzy objectoriented databases using extended entityrelationship model. International Journal of Intelligent Systems, 16, 697-711.

[20] Marín, N., Vila, M. A., \& Pons, O. (2000). Fuzzy types: A new concept of type for managing vague structures. International Journal of Intelligent Systems, 15, 1061-1085.

[21] Ma, Z. M., Zhang, W. J., \& Ma, W. Y. (2004). Extending object-oriented databases for fuzzy information modeling. Information Systems, 29(5), 421-435.

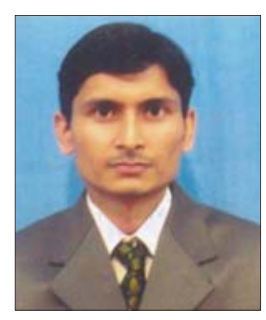

Mr.Praveen Shukla - Senior faculty member -Department of Information Technology, Northern India Engineering College, Lucknow. His area of research is Fuzzy Object Oriented Database. He has presented several papers in national and international conferences. 